\title{
Prehabilitation and its relevance to patient outcome
}

\author{
Dr Loranthi Samarasinghe ${ }^{1}$ \\ Consultant Anaesthetist, Lanka Hospitals, 578, Elvitigala Mawatha, Colombo 5, Sri Lanka.
}

Prehabilitation is defined as the process of enhancing an individual's functional capacity to enable him or her to withstand a forthcoming stressor e.g. major surgery. ${ }^{1}$ Its use in sports refers to the form of strength training required to prevent injuries before they happen and has the origin from the words "pre"+ "rehabilitation" and is probably the source from where the word moved into medical jargon. In medical practice prehabilitation refers to the process of "enhancing a patient's functional capacity before surgery with the aim of improving post-operative outcome". ${ }^{1}$

Improving surgical outcome, following major surgery, especially in the high-risk patient, who is elderly with multiple comorbidities, remains a challenge. In the UK this group accounts for $80 \%$ of the post-operative deaths although they are only $12.5 \%$ of the surgical admissions. ${ }^{1}$ Sri Lankan statistics are not available although it is reasonable to assume that they are similar or worse.

Pre-operative optimization has a long history in anaesthetic practice and relates to the improvement of a patient's medical parameters prior to surgery. However, the use of the word prehabilitation refers to a broader concept, where medical optimization, physical exercise, nutritional support, psychological support and behavior modification, is practiced over a minimum of 4 weeks before undertaking surgery.

*Correspondence: Loranthi Samarasinghe
E mail: loranthies@gmail.com

https://orcid.org/0000-0002-5554-9397 DOI: http:/doi.org/10.4038/slja.v28i1.8535

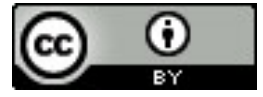

Medical optimization is currently practiced universally during the pre anaesthetic assessment that is performed prior to surgery. Its benefits are well documented and understood. The management of anaemia, control of blood pressure and blood glucose too has shown to improve post operative outcome and are a part of the routine aspects looked at traditionally in the pre anaesthetic assessment.

Pre-operative exercise programmes are a unique aspect of prehabilitation.

Improving physical fitness is a logical step to achieve improved outcome after surgery. It is directed at improving endurance, strength and respiratory muscle function. Physical exercise programs have been drawn up to improve a patient's functional capacity prior to elective surgery. Physical exercise testing can be in the form of CPET (cardiopulmonary exercise testing) or the 6 minute walk test. These tests are repeated over a period of time to establish improvement in physical fitness following exercise training. Exercise training is undertaken to reach the Bory scale target of $12-16$. The Bory scale is used to assess the strenuousness of exercise. ${ }^{2}$

Referral for prehabilitation should be performed after a thorough assessment of the risks related to the patient's medical and physical status. Appropriate referral with adequate time for intervention - minimum of 4 weeks - must be undertaken and the goals of the programme must be clearly identified and the patient given adequate written instructions regarding the whole process.

Several studies have looked at physical exercise training prior to a variety of surgeries i.e lung resection in non small cell carcinoma of the lung, major abdominal surgery etc. The outcomes looked at were post-operative pulmonary complications, post-operative pulmonary catheter 
usage, post-operative mortality and postoperative hospital and ICU stays. Due to heterogeneity of the groups studied, small sample sizes and the lack of standardization of outcomes measured, there has been a lack of conclusive evidence regarding its benefits. ${ }^{2}$ However this seems to be more due to the lack of adequate RCTs as opposed to the futility of the procedure.

Behavior modification and psychological support is the $3^{\text {rd }}$ aspect of prehabilitation. The benefits of cessation of smoking is well established where the nicotine and carbon monoxide levels in the blood reduce significantly even with short durations of abstinence. ${ }^{3}$ Following abstinence for 24 to 48 hours the deleterious effects dissappear. ${ }^{1}$ Longer periods of abstinence restore ciliary function and reduce bronchiolar irritation significantly. Trials that looked at 4 weeks of cessation of smoking had a significantly larger effect on perioperative mortality and morbidity. ${ }^{3}$ A randomized control trial on 42 patients showed that pre-operative abstinence from alcohol has a beneficial effect with regards to reduced immunosuppression, postoperative hypoxaemia, myocardial infarctions and arrhythmias. ${ }^{4}$

Nutritional support in the form of weight gain or weight loss, as required, addresses the final aspect of prehabilitation. The benefits are obvious.

Prehabilitation in spite of the lack of conclusive evidence is here to stay. Its appropriateness rests on the proper identification and adequate, timely institution of tailor made programmes for each individual patient. This process requires coordination and planning for efficient and effective functioning and would contribute to further minimizing post-operative morbidity and mortality.

\section{References}

1. Banugo p, Amoako D. Prehabilitation. BJA Education, 2017; 17(12): 401-405 https://doi.org/10.1093/bjaed/mkx032

2. Tew G A, Ayyash R, Durrand J, Danjoux G R. Clinical guideline and recommendations on preoperative exercise training in patients awaiting major non cardiac surgery. Anaesthesia, 2018; 73:750-768.

https://doi.org/10.1111/anae.14177
3. Carrick M A, Robson J M, Thomas C.Smoking and anaesthesia, BJA Education, 2019; (1): 1-6 https://doi.org/10.1016/j.bjae.2018.09.005

4. Tonnesen $\mathrm{H}$, Rosenberg J, Nielsen $\mathrm{H} \mathrm{J}$ et al, Effect of pre-operative abstinence on poor post operative outcome in alcohol misusers: randomized controlled trial. BMJ,1999; 15;318 (7194):1311-6 https://doi.org/10.1136/bmj.318.7194.1311 